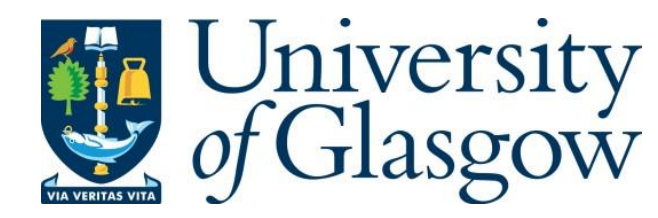

Liu, Y., Huang, Y., Zhang, R., Hou, L., Lu, D., Zhao, L. and Wang, W. (2022) Optical and RIN spectrum improvements in necked waveguide high-power DFB laser diode. IEEE Photonics Technology Letters, (doi: 10.1109/LPT.2022.3150799).

There may be differences between this version and the published version. You are advised to consult the publisher's version if you wish to cite from it.

https://eprints.gla.ac.uk/265063/

Deposited on: 9 February 2022

Enlighten - Research publications by members of the University of Glasgow https://eprints.gla.ac.uk 


\title{
Optical and RIN Spectrum Improvements in Necked Waveguide High-Power DFB Laser Diode
}

\author{
Yihui Liu, Yongguang Huang, Ruikang Zhang, Lianping Hou, Senior Member, IEEE, Dan Lu, Lingjuan Zhao, Wei
} Wang, Member, IEEE

\begin{abstract}
A novel high power distributed feedback (DFB) laser is proposed which uses a partially narrow waveguide and corrugation pitch modulated (CPM) phase shift grating. Compared with conventional DFB lasers with constant waveguide width, these necked DFB lasers have greater improvements in terms of optical and relative intensity noise (RIN) spectrum. They are less sensitive to external optical feedback (EOF) and increase the range of the injection current for single longitudinal mode (SLM) operation. It can not only suppress the multimode lasing state under high injection current and EOF intensity, but also realize the SLM operation with RIN < -135 dB under a EOF intensity of less than $-15 \mathrm{~dB}$ in the current range of $150 \mathrm{~mA} \sim 350$ $\mathrm{mA}$. Therefore, the range of injection current for SLM operation was increased for continuous wave $(\mathrm{CW})$ light sources in siliconbased hybrid photonic integration applications.
\end{abstract}

Index Terms - coherent collapse, relative intensity noise (RIN), external optical feedback, high power DFB, continuous wave (CW), necked waveguide, corrugation pitch modulated (CPM).

\section{INTRODUCTION}

$\mathrm{H}$ IGH-power unilateral-lasing continuous wave $(\mathrm{CW})$ distributed feedback (DFB) lasers with external optical coupling has great application potential as light sources for silicon-based hybrid photonic integration. However, the external optical coupling will introduce unexpected reflections which can easily occur when there are refractive index discontinuities along the transmission direction [1]. In many circumstances, external optical feedback (EOF) from an external cavity may have a profound impact on the optical spectral linewidth and dynamic behavior of laser diodes (LDs) [2]. Several theoretical and experimental investigations on LD performances due to EOF have been performed before [3-6]. For stable single longitudinal mode (SLM) and superior lownoise laser operation, an expensive optical isolator is usually introduced and cascaded to the emitting face of the

This work was supported by the National Natural Science Foundation of China under Grant 62074141 and by the U.K. EPSRC (EP/R042578/1) (Corresponding author: Yongguang Huang.)

Yihui Liu, Yongguang Huang, Ruikang Zhang, Dan Lu, Lingjuan Zhao, Wei Wang are with the Key Laboratory of Semiconductor Materials Science, Institute of Semiconductors, Chinese Academy of Sciences, Beijing 100083, China, also with the Center of Materials Science and Optoelectronics Engineering, University of Chinese Academy of Sciences, Beijing 100049, China, and also with the Beijing Key Laboratory of Low Dimensional Semiconductor Materials and Devices, Beijing 100083, China (e-mail: yghuang@ semi.ac.cn).

Lianping Hou is with the James Watt School of Engineering, University of Glasgow, Glasgow G12 8QQ, U.K. (email:Lianping.Hou@glasgow.ac.uk)
DFB laser to eliminate the EOF [7]. But this is not feasible in silicon-based hybrid photonic integration since most optical isolators cannot be monolithically fabricated. Therefore, one of the research topics is to design waveguide-based optical isolators capable of being integrated with LDs [8]. Another research direction is to develop DFB LDs which improve the resistance characteristics to EOF, expand the range of injection current for stable SLM of the isolator-free DFB LDs. Several methods have been reported, such as gain/complex- coupled DFB lasers [9], partially corrugated grating LDs (PC-LD) [10], quantum dot (QD) loss-coupled DFB laser with HR/HR coated facets [11], interband cascade lasers [12], and multi-period partially tilted grating FP lasers [13] etc. In this paper, we proposed a high-power DFB laser with high immunity to EOF. The proposed device is formed by inserting a section of narrow width waveguide in a conventional straight waveguide device, which is named as neck-ridged waveguide here. Within the narrow waveguide, there is a corrugation pitch modulated (CPM) phase-shift grating section. For comparison, we also proposed a straight waveguide CPM-DFB (S-CPM-DFB) laser with the same grating structure and cavity length fabricated on the same epitaxial layer. By comparing the performances in optical spectrum and relative intensity noise (RIN) of these two devices at the same EOF intensity, we found the necked waveguide with CPM grating DFB (N-CPM-DFB) laser had smaller sensitivity to EOF and the RIN resonance peaks are greatly reduced. The N-CPM-DFB LDs fabrication process is compatible with conventional DFB LDs, significantly reducing fabrication complexity and cost.

EOF is mainly considered as an external interference that affects the laser optical linewidth and noise level. LDs are intrinsically noisy devices because of the quantum nature of light. Even when biased at a constant current, the LD exhibits fluctuations in its phase and intensity due to temperature and aging. The effect of EOF is determined by many factors, such as feedback intensity, external reflected round-trip time, injection current and end facet reflectivity, etc. [1-3]. The EOF can lead to LD intensity and phase fluctuations, broaden or reduce the optical linewidth, and accordingly deteriorate or improve the signal-to-noise ratio (SNR) performance. These can be characterized by the optical and RIN spectrum [14]. When the EOF is less than $-50 \mathrm{~dB}$, the effect of the EOF phase on the spectral linewidth can be ignored [3]. Therefore, we mainly focus on the EOF intensity with a range of $-10 \mathrm{~dB} \sim$ $-50 \mathrm{~dB}$. 


\section{DEVICE STRUCTURE AND MEASUREMENT SETUP}

The waveguide and grating structure schematic diagrams of the S-CPM-DFB and N-CPM-DFB lasers are shown in Fig. 1. They both have the same cavity length of $1000 \mu \mathrm{m}$ with antireflective (AR) coating $(0.12 \%)$ and high-reflective (HR) $(98.74 \%)$ coated on the facets of the devices. The active region is composed of AlGaInAs strained multiple quantum wells (MQWs) with a center wavelength of around $1310 \mathrm{~nm}$. The uniform gating period was $203.5 \mathrm{~nm}$ and the CPM grating period was $203.6 \mathrm{~nm}$ which was used to produce effective $\lambda / 4$ phase shift ( $\lambda$ denotes the Bragg wavelength), both of which are defined by electron beam lithography (EBL) and reactive ion etching (RIE) process. For the N-CPM-DFB laser, the necked waveguide is $100 \mu \mathrm{m}$ long and overlaps with CPM grating segment with $5 \mu \mathrm{m}$ long tapered section at both sides of the necked section. The narrowed ridge segment is $2.1 \mu \mathrm{m}$ wide with a grating coupling coefficient $\kappa$ around $14.6 / \mathrm{cm}$, and the other sections are $2.5 \mu \mathrm{m}$ wide with a $\kappa$ value around $17 / \mathrm{cm}$. The S-CPM- DFB laser has the same grating layout but with a constant waveguide width of $2.5 \mu \mathrm{m}$ with a $\kappa$ value around $17 / \mathrm{cm}$.

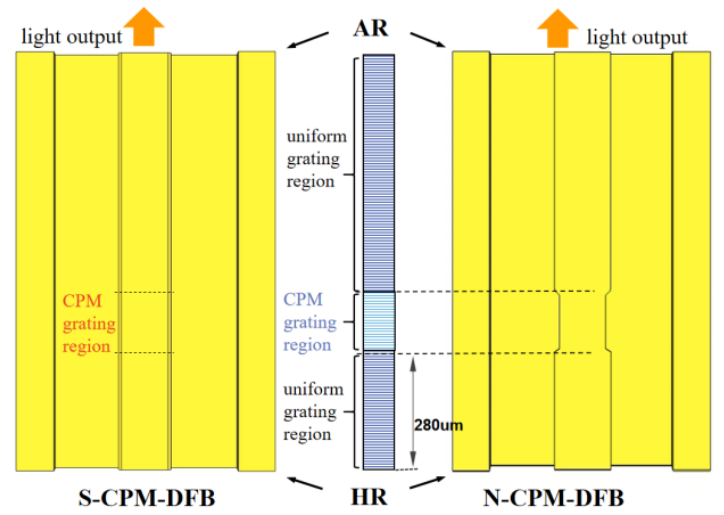

Fig. 1. Schematical diagram of S-CPM-DFB LD (left) and N-CPM-DFB LD (right) waveguide and grating structures.

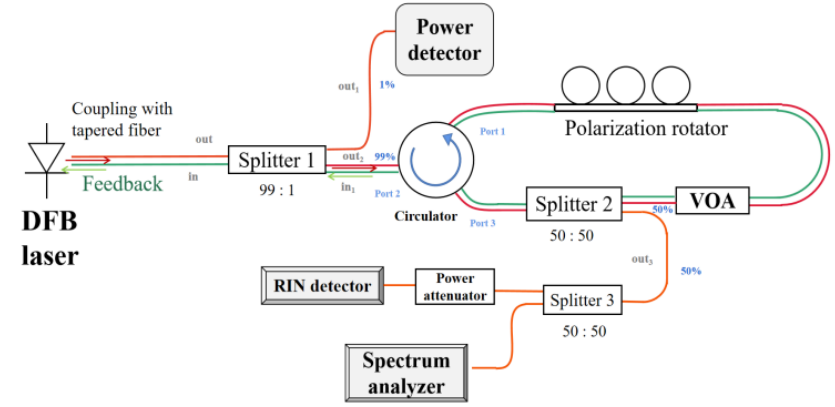

Fig. 2. Schematic diagram of tunable feedback optical circuit testing setup.

Fig. 2 shows the setup to tune the intensity of the EOF and measure the optical spectrum and RIN of the devices with EOF. The test LDs was stuck onto the chip carrier with a temperature control (TEC) stage underneath $\left(25 \mathrm{C}^{\circ}\right)$. The output light of the LD was coupled to a tapered optical fiber and then entered to Splitter 1 which has 1\% light entering the power meter and $99 \%$ entering the circulator. The optical power-meter was used for real-time measurement of the total optical power coupled into the optical fiber. The light from port 3 of the circulator then entered Splitter 2 which evenly divided the input light into two parts, one for optical feedback loop, and another one for multiparameter measurements, such as optical spectrum and RIN. Since the system was dynamically balanced, the light output from the circulator is under a EOF modulated state. The feedback ratio was controlled by using a variable optical attenuator (VOA). A polarization controller (PC) was used to adjust the polarization of the feedback light to obtain the maximum feedback effect under a given feedback level to reach the worst situation for the laser. The lowest RIN floor of the measurement machine is around $-150 \mathrm{~dB}$, and the RIN detector maximum input power should not exceed $3 \mathrm{dBm}$, therefore an adjustable attenuator was placed in front of RIN detector. Eq. (1) was used to calculate EOF intensity of the system:

$$
A_{\text {system }}=A_{\text {coupling }} \times 2+A_{\text {splitter } 1}+A_{\text {splitter } 2}+A_{V O A}
$$

$A_{\text {system }}$ is the total intensity of the system return optical feedback; $A_{\text {splitter } 1}$ and $A_{\text {splitter } 2}$ are the losses of the beam Splitter 1 and Splitter 2, which were fixed values. $A_{V O A}$ is the tunable loss introduced by the VOA; $A_{\text {coupling }}$ is the coupling loss between the LDs and the tapered fiber which make sure it a constant value of $-3.5 \mathrm{~dB}$. The whole EOF round-trip loop length is 130 $\mathrm{cm}$ with a fixed delay time of $6.4 \mathrm{~ns}$.

\section{EXPERIMENTAL RESULTS AND ANALYSIS}

At $25^{\circ} \mathrm{C}$, the threshold currents of S-CPM-DFB and N-CPMDFB are $29.5 \mathrm{~mA}$ and $26.9 \mathrm{~mA}$ respectively. Under $400 \mathrm{~mA}$, their output powers are $97.2 \mathrm{~mW}$ and $97.4 \mathrm{~mW}$, respectively. For comparison, the optical and RIN spectra were measured for the $1 \mathrm{~mm}$ long DFB LD with a uniform grating and an abrupt $\lambda / 4$ phase shift at $330 \mu \mathrm{m}$ from the HR coated facet, which was fabricated using the same wafer and same facets' reflectivity.

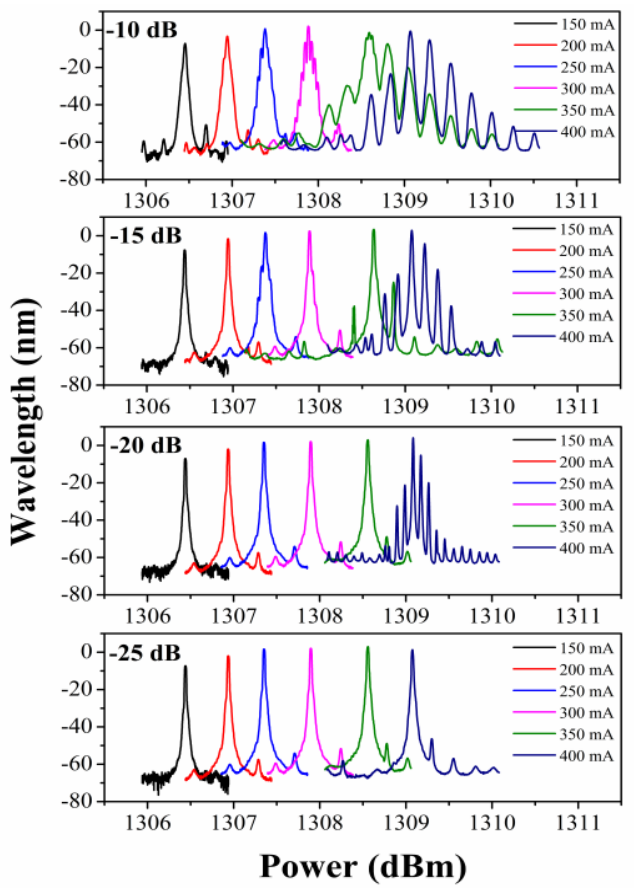

(a) 


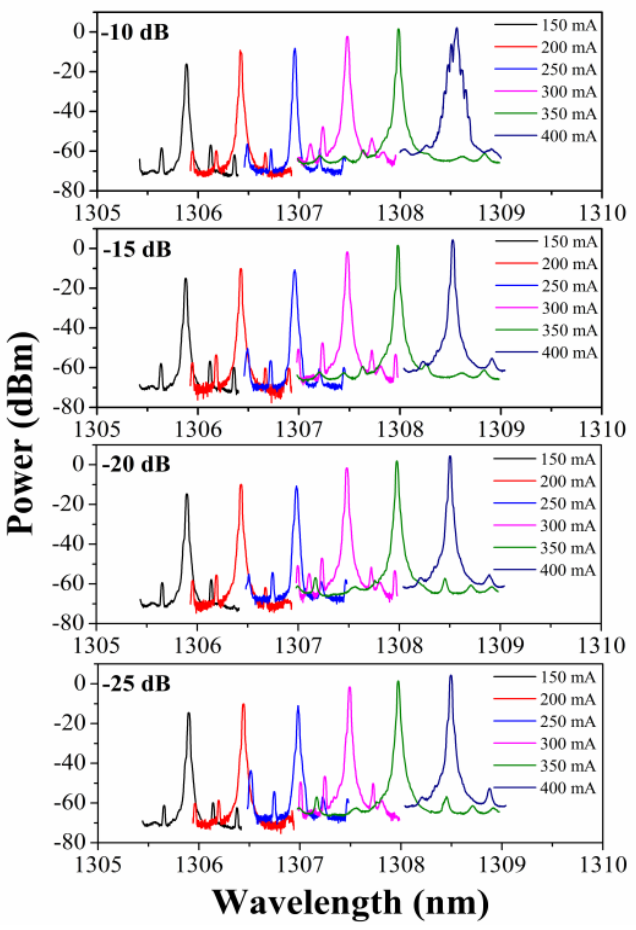

(b)

Fig. 3. Spectrum at a current of $150 \mathrm{~mA} \sim 400 \mathrm{~mA}$ under $-10 \mathrm{~dB} \sim-25 \mathrm{~dB}$ EOF intensity for (a) S-CPM-DFB LD, and (b) N-CPM- DFB LD.
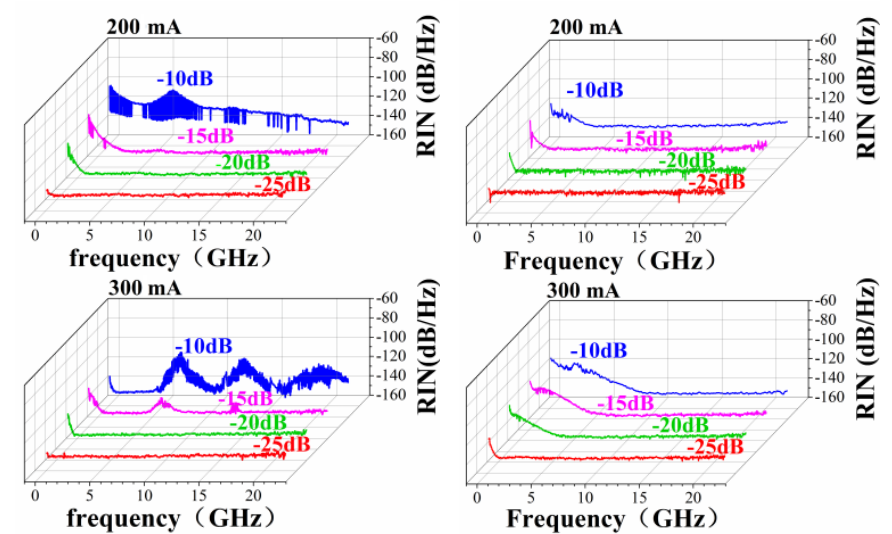

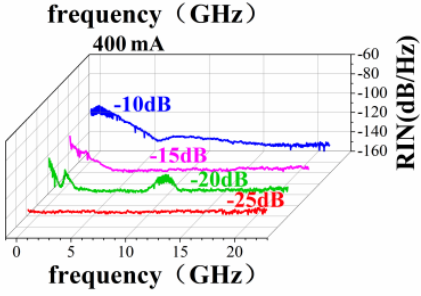

(a)

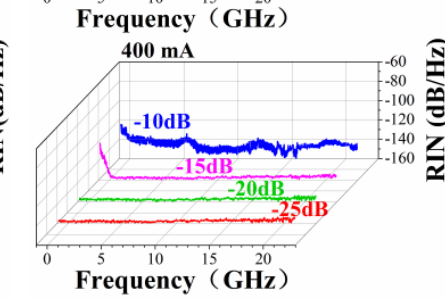

(b)
Fig. 4. RIN at a current of $200 \mathrm{~mA}, 300 \mathrm{~mA}, 400 \mathrm{~mA}$ under $-10 \mathrm{~dB} \sim-25 \mathrm{~dB}$ EOF intensity for (a) S-CPM-DFB LD, and (b) N-CPM-DFB LD.

From Fig.3 (a) and Fig.4 (a), S-CPM-DFB LD can only achieve stable SLM from $150 \mathrm{~mA}$ to $300 \mathrm{~mA}$ under less than $25 \mathrm{~dB}$ EOF intensity. When strong feedback $(-10 \mathrm{~dB})$ was applied to the S-CPM-DFB LD, it transited a stable singlemode and then transited to a coherent collapse mode [5] when the injection current was increased which initiated multi-mode lasing at a current of more than $350 \mathrm{~mA}$. The noise and linewidth of the device was further deteriorated [16]. In the coherent collapse region, the LD tended to be unstable, behaving as a wide emission spectrum and having a considerable frequency fluctuation and increasing the overall noise intensity which was confirmed by increasing $20 \mathrm{~dB}$ in the RIN level of the relaxation oscillation peak (much pronounced in Fig.4(a) at $300 \mathrm{~mA}$ ). The RIN curve contains multiple oscillation peaks, and their frequencies correspond to resonance frequency $6.18 \mathrm{GHz}$ and its multiple value [15]. When decreasing the EOF intensity, the DFB laser can maintain a stable SLM operation. At -20 dB EOF intensity, the LD did not transit to the coherent collapsed state until an injection current of $400 \mathrm{~mA}$, where the resonant peak of the RIN appears around the double resonance frequency, i.e. $12.34 \mathrm{GHz}$. As the EOF intensity was further reduced, such as below $-25 \mathrm{~dB}$, the LD was able to keep the SLM operation stable in the injection current range of $150 \mathrm{~mA} \sim 400 \mathrm{~mA}$, with RIN $<-140 \mathrm{~dB}$. The effect of the EOF to the S-CPM-DFB was much more pronounced under a larger bias current. It even entered the multi-mode lasing state under high injection current range, which severely limited the effective operating current range in practical application.

In comparison, even under a strong EOF of $-10 \mathrm{~dB}, \mathrm{~N}-\mathrm{CPM}-$ DFB laser will not be deteriorated to a multi-mode lasing state (see Fig.3(b) and Fig.4(b)). Even under high injection current, its critical current value for coherent collapse has also improved significantly. Within the current range of $150 \mathrm{~mA} \sim 350 \mathrm{~mA}$, the optical spectrum keeps a SLM with side mode suppression ratio (SMSR) > $45 \mathrm{~dB}$. As the laser power increases, the feedback intensity range corresponding to the coherent collapse region became smaller. This can be qualitatively understood since the instability is driven by spontaneous emission and the effects of EOF noise are reduced when laser power increases, due to improved photon statistics. In addition, the photon distribution along the cavity has been improved due to the introduction of the necked waveguide, the effect of EOF noise is reduced when the laser power is increased. Apart from this, N-CPM-DFB laser can quickly move to a stable SLM state as the EOF intensity decreases. When EOF is below $-15 \mathrm{~dB}$, the $\mathrm{N}-\mathrm{CPM}-\mathrm{DFB}$ laser has a SLM operation with SMSR $>40 \mathrm{~dB}$ in the current range of $150 \mathrm{~mA} \sim 400 \mathrm{~mA}$, and the RIN is less than $-142 \mathrm{~dB}$.
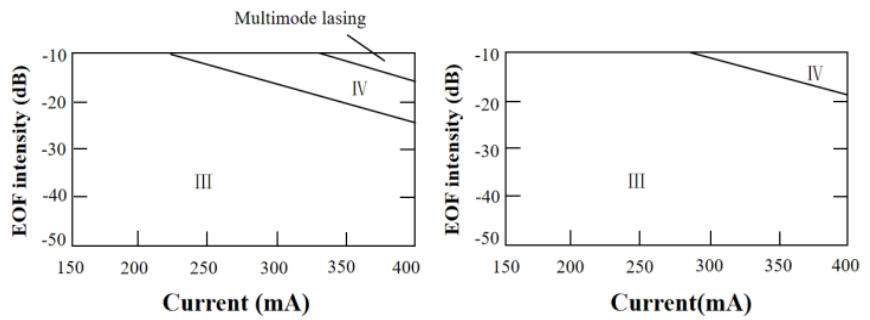

Fig. 5. The optical mode map (a) S-CPM-DFB LD, and (b) N-CPM-DFB LD in the current range of $150 \mathrm{~mA} \sim 400 \mathrm{~mA}$ and the EOF intensity of $-10 \mathrm{~dB} \sim-$ $50 \mathrm{~dB}$.

By comparing the two types of DFB lasers, we found that NCPM-DFB lasers have better resistance to EOF than that of SCPM-DFB. Fig. 5 shows the lasing mode maps as a function of the injection current and the intensity of the EOF. Among them, 
III is a SLM area, and IV represents the coherent collapse area. It is obvious that the N-CPM-DFB laser has a wider range of SLM area than that of the S-CPM-DFB LD and did not show the multimode lasing state even under $400 \mathrm{~mA}$ and $-10 \mathrm{~dB}$ EOF.

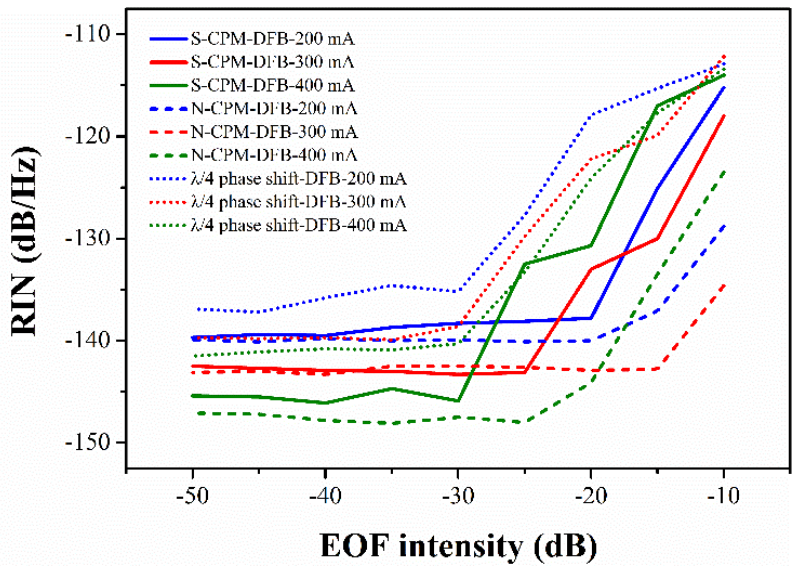

Fig. 6. RIN of S-CPM- DFB, N-CPM- DFB , and $\lambda / 4$ phase shift DFB LDs at a current of $200 \mathrm{~mA}, 300 \mathrm{~mA}, 400 \mathrm{~mA}$ when the EOF intensity was changed from $-10 \mathrm{~dB}$ to $-50 \mathrm{~dB}$ in steps of $-5 \mathrm{~dB}$.

Fig. 6 shows the RIN curves versus the EOF intensity for the two types of lasers with injection current from $200 \mathrm{~mA}$ to 400 $\mathrm{mA}$. The N-CPM-DFB laser has better characteristics of suppressing the increase of RIN value under higher external light feedback, especially under higher current. For comparison, the RIN curves versus the EOF intensity of the $1 \mathrm{~mm}$ long abrupt $\lambda / 4$ phase shift DFB LD were also shown, where the device was much sensitive to the EOF.

The primary source of the intensity noise in the LD is the spontaneous emission that is usually amplified due to the external feedback effect. The joint effect of the internal and external cavity is based on optimizing the resonance between photons and carriers to suppress RIN [15]. For N-CPM-DFB lasers, the introduction of a necked waveguide in the CPM section leads to less sensitivity to the effect of the CPM phase mismatching and didn't reduce the effective grating coupling coefficient. Therefore, the intensity distribution along the cavity is flatter and the spatial hole burning effect is alleviated, reducing the strong effect of the EOF in the entire cavity.

Many studies have shown that the feedback sensitivity of the lasers was evaluated from the stability of the longitudinal mode shape, the optical field depends only on the phase of the HR facet [9]. Therefore, the necked waveguide is designed near the HR facet, which more efficiently suppresses the uneven distribution of photons and carriers. The N-CPM-DFB LD can be further optimized, such as the waveguide width of the necked section, the length of the necked section, the distance between the necked section and the HR surface, the grating duty ratio of the CPM section, etc. A more robust N-CPM-DFB LD could be achieved even under larger EOF intensity.

\section{CONCLUSION}

A robust N-CPM-DFB LD was proposed and characterized in terms of the optical and RIN spectrum by a tunable feedback optical circuit test system. Compared with the S-CPM-DFB LDs, the necked waveguide combined with the CPM phase shift grating increased resistance to EOF. It can not only suppress the multimode lasing state under high injection current and strong EOF, but also realize the SLM operation with RIN $<-135 \mathrm{~dB}$ under the EOF intensity of less than $-15 \mathrm{~dB}$ in the current range of $150 \mathrm{~mA} \sim 350 \mathrm{~mA}$. Therefore, it will increase the range of the injection current for SLM operation as CW light sources for silicon-based hybrid photonic integration application.

\section{REFERENCES}

[1] N. Schunk and K. Petermann, "Numerical analysis of the feedback regimes for a single-mode semiconductor laser with external feedback," IEEE Journal of Quantum Electronics, vol. 24, no. 7, pp. 1242-1247, July 1988.

[2] A. Jha, M. K. Shah, S. Jha, L. R. Cenkeramaddi and S. Royo, "Current Modulation Induced Stability in Laser Diode Under High Optical Feedback Strength," in IEEE Access, vol. 9, pp. 49537-49546, 2021.

[3] R. Tkach and A. Chraplyvy, "Regimes of feedback effects in $1.5-\mu \mathrm{m}$ distributed feedback lasers," in Journal of Lightwave Technology, vol. 4, no. 11, pp. 1655-1661, November 1986.

[4] Espinola, R. L., et al. "Magneto-optical nonreciprocal phase shift in garnet/silicon-on-insulator waveguides." Optics Letters, vol. 29, no. 9, pp. $941,2004$.

[5] D. Lenstra, B. Verbeek and A. Den Boef, "Coherence collapse in singlemode semiconductor lasers due to optical feedback," IEEE Journal of Quantum Electronics, vol. 21, no. 6, pp. 674-679, June 1985.

[6] Yidong Huang, T. Okuda, K. Shiba and T. Torikai, "High-yield external optical feedback resistant partially corrugated waveguide laser diodes," in IEEE Journal of Selected Topics in Quantum Electronics, vol. 5, no. 3, pp. 435-441, May-June 1999.

[7] Turner, A. E., R. L. Gunshor, et al. "New class of materials for optical isolators." Applied Optics, vol. 22, no. 20,pp. 3152-3514,1983.

[8] Bi, Lei, et al. "On-chip optical isolation in monolithically integrated nonreciprocal optical resonators." Nature Photonics, vol. 5. no. 12, pp. 758762, Dec. 2011.

[9] Y. Nakano, Y. Deguchi, K. Ikeda, Y. Luo and K. Tada, "Reduction of excess intensity noise induced by external reflection in a gain-coupled distributed feedback semiconductor laser," IEEE Journal of Quantum Electronics, vol. 27, no. 6, pp. 1732-1735, June 1991.

[10]M. Gotoda, T. Nishimura, K. Matsumoto, T. Aoyagi and K. Yoshiara, "Highly External Optical Feedback-Tolerant $1.49 \mu \mathrm{m}$ Single-Mode Lasers With Partially Corrugated Gratings," IEEE Journal of Selected Topics in Quantum Electronics, vol. 15, no. 3, pp. 612-617, May-june 2009.

[11]H. Su et al., "External feedback effects on a quantum dot laterally loss coupled distributed feedback laser," The 16th Annual Meeting of the IEEE Lasers and Electro-Optics Society, 2003. LEOS 2003., 2003, pp. 198-199 vol.1.

[12]Y. Deng, Z. -F. Fan and C. Wang, "Optical Noise of Interband Cascade Lasers Subject to Optical Feedback," 2020 IEEE Photonics Conference (IPC), 2020, pp. 1-2.

[13]Y. Pan, Y. Xi and X. Li, "Detuned Grating Single-Mode Laser With High Immunity to External Optical Feedback," IEEE Photonics Journal, vol. 7, no. 6, pp. 1-13, Dec. 2015, Art no. 1504013, doi: 10.1109/JPHOT.2015. 2497581.

[14]Y. Hong, S. Bandyopadhyay, S. Sivaprakasam, P. S. Spencer and K. A. Shore, "Noise characteristics of a single-mode laser diode subject to strong optical feedback," Journal of Lightwave Technology, vol. 20, no. 10, pp. 1847-1850, Oct. 2002.

[15]K. I. Kallimani and M. J. O'Mahony, "Relative intensity noise for laser diodes with arbitrary amounts of optical feedback," IEEE Journal of Quantum Electronics, vol. 34, no. 8, pp. 1438-1446, Aug. 1998.

[16] H. Li, J. Ye and J. G. McInerney, "Detailed analysis of coherence collapse in semiconductor lasers," IEEE Journal of Quantum Electronics, vol. 29, no. 9, pp. 2421-2432, Sept. 1993. 